\title{
Genetic evolution during tumor progression: from basic research to clinical practice
}

\author{
József Tímár ${ }^{1,2}$
}

Published online: 10 March 2016

(C) Springer Science+Business Media New York 2016

Most of the primary cancers of various stages are resected surgically or destroyed by radiotherapy except for lung cancers and hematopoetic cancers. Contemporary molecularly targeted therapies are administered in advanced stages of cancer but the required molecular pathologic analysis is performed mostly on the primary tumor, assuming stable genetics during cancer progression. One of the hallmarks of cancer is genomic instability leading to clonal heterogeneity of not only the primary but also the metastatic tumors as well. This clonal heterogeneity is reflected both in the founder driver mutations as well as in the later branched events. Based on data from experimental systems, three competing models of genetic evolution exist: the same gene, the independent/parallel evolution, and the clonal progression models. Genetic consequences of the validity of these models are completely different. In case of the same gene model metastasis(es) is/are highly comparable to the primary tumor, in case of the parallel model metastasis(es) is/are uncomparable, while in case of the clonal model genetics of the metastatic clones show match only to a small subpopulation of the primary tumor. It is most probable that in various cancers and progression forms (locoregional lymphatic progression, synchronous hematogenous dissemination, metachronous hematogenous dissemination), one or another model plays a predominant role. The current special issue of Cancer and Metastasis Reviews is devoted to this theoretical and clinically highly practical question. In this

József Tímár

jtimar@gmail.com

1 2nd Department of Pathology, Semmelweis University, Ülloii u. 93, Budapest H-1091, Hungary

2 Molecular Oncology Research Group, Hungarian Academy of Sciences, Semmelweis University, Budapest, Hungary issue, the problem will be covered from two aspects: a smaller experimental part that deals with the analysis of tumor models to show how the genetics changes from primary tumor to metastases in the circulation or in various organs (Hoffmann), and how stemness affects this problem (Bodenstine et al.). Major part of the issue is devoted to this question from clinical and pathological points of view: how do the genetics of primary tumors change in various cancer types during metastatization, are there enough data to address this issue, and are there any metastatic organ-specific features in this respect? Hematogenous dissemination of cancer is manifested in the presence of cancer cells in the circulation called circulating cancer cells (CTCs), and this hot issue getting more and more clinical attention today is addressed by a long-time pioneer, Joosse and Pantel. The emergence of liquid biopsy technology is related to, but not equal with this problem. Genetic progression of breast cancer is one of the best described examples of clonal selection, genetic instability, and induced genetic alterations during metastatic progression, documented and commented by Desmedt et al. With the advent of molecular targeted therapy of colorectal cancer, genetic progression during metastatization has become a hot issue, which is nicely summarized by Amaro et al. Lung cancer is unique among solid tumors in a sense that in the majority of cases, metastatic progression occurs in the presence of the primary tumor and the locoregional lymphatic metastases providing a continuous rich source for intravasating tumor cells. On the other hand, unlike most solid cancers, primary lung cancer is highly heterogeneous morphologically (as well as genetically). This complexity is perfectly emphasized by Popper. Malignant melanoma is one of the most metastatic human cancer types reflected clearly by the log smaller T size parameters $(\mathrm{mm})$ as compared to other solid tumors $(\mathrm{cm}$ range). Furthermore, malignant melanoma has one of the most highly mutated genomes as well. Data are starting to 
accumulate for this cancer type in respect to how metastatic progression is reflected at genomic level and also how novel target or immunotherapies influence these processes (Tímár et al.). Hematopoetic malignancies, including lymphomas, have long been considered as mono- or oligoclonal diseases, but lately, it has been discovered that they are highly similar to other solid malignancies in this respect as well. Their genetic progression, especially the treatment-induced forms, is described in detail by Bödör and Reiniger. At the end of the issue, a respected molecular pathologist gives us an analysis of this question from the viewpoint of everyday molecular diagnostics (Ryska), and an internationally recognized oncologist analyzes the problem clinically (Pyrker): how this new knowledge would affect treatment decisions, trial designs, or guidelines in case of lung cancer patient management. These papers all clearly demonstrate that cancer, especially in its progressing forms, is a continuously moving target due not only to the imminent genetic features but also more and more likely due to the environmental genetic pressures provided by chemo-, targeted-, and current immunotherapies. It is better to face this challenge, discover its biological basis rather than blindly hope for a theoretical stability. 